\section{Conceptos actuales de sepsis y shock séptico}

Current concepts of sepsis and septic shock

\section{RESUMEN}

La sepsis es una causa principal de enfermedad crítica y mortalidad hospitalaria. El reconocimiento temprano y la intervención son esenciales para la supervivencia de los pacientes. El objetivo de esta investigación es actualizar la información disponible sobre sepsis y shock séptico. El análisis documental se realizó empleando motores de búsqueda avanzada de información científica relacionada a la salud, como Pubmed, Imbiomed y Scielo, seleccionando artículos de del 2011 al 2019 mediante el uso de descriptores de la salud y los medical subject headings. La muestra quedo conformada por un total de 18 artículos científicos de revisión de la literatura y 5 artículos de investigación analítica. Se revisó la definición, epidemiología, fisiopatología, enfoques diagnósticos y terapéuticos de la sepsis y el shock séptico.

PALABRAS CLAVE: sepsis, shock séptico, biomarcadores, SOFA, fisiopatología.

\section{ABSTRACT}

Sepsis is a leading cause of critical illness and hospital mortality. Early recognition and intervention are essential for patient survival. The objective of this research is to update the available information on sepsis and septic shock. The documentary analysis was performed using advanced search engines of scientific information related to health, such as Pubmed, Imbiomed and Scielo, selecting articles from 2011 to 2019 through the use of health descriptors and medical subject headings. The sample was made up of a total of 18 scientific literature review articles and 5 analytical research articles. The definition, epidemiology, pathophysiology, diagnostic and 
therapeutic approaches of sepsis and septic shock were reviewed.

KEYWORDS: sepsis, septic shock, biomarkers, SOFA, pathophysiology.

\section{INTRODUCCIÓN}

La sepsis es una emergencia médica que describe la respuesta inmunológica sistémica del cuerpo a un proceso infeccioso que puede conducir a la disfunción orgánica y la muerte. A pesar de los avances significativos en la comprensión de la fisiopatología de este síndrome clínico, los avances en las herramientas de monitoreo hemodinámico y las medidas de reanimación, la sepsis sigue siendo una de las principales causas de morbilidad y mortalidad en pacientes críticos. La incidencia anual de sepsis severa y shock séptico en los Estados Unidos es de hasta 300 casos por cada 100,000 personas (1).

Sin embargo, la carga epidemiológica global de la sepsis es difícil de determinar. Se estima que más de 30 millones de personas se ven afectadas por sepsis cada año en todo el mundo, lo que resulta en un potencial de 6 millones de muertes al año (Cecconi M, 2018). Las tasas de mortalidad por sepsis, según los datos de Surviving Sepsis Campaign 2012, fueron aproximadamente del $41 \%$ en Europa frente a aproximadamente el $28,3 \%$ en los Estados Unidos (3). Sin embargo, esta diferencia desapareció cuando se ajustó la gravedad de la enfermedad. Esto implica que la mortalidad en la sepsis también varía según las características del paciente.

El objetivo de esta investigación es actualizar la información disponible sobre sepsis y shock séptico. Se revisó el historial de sepsis y las definiciones de afecciones relacionadas desde el primer consenso en 1991 hasta la actualidad, los datos epidemiológicos resultantes de los estudios mundiales sobre incidencia y mortalidad, enfoques de diagnóstico que incluyen la evaluación microbiológica de la infección, uso de varias escalas pronósticas, biomarcadores de diagnóstico.

\section{MATERIALES Y MÉTODOS}

Se realizó una búsqueda sistemática en la base de datos de PubMed, Imbiomed y Scielo del 1 enero del 2011 hasta el 31 de diciembre del 2019, excepto estudios relevantes de carácter histórico que deben ser mencionados en este estudio. Se incluyeron artículos de idioma inglés y español, relacionados con la definición, epidemiología, diagnóstico y fisiopatología de la sepsis y shock séptico. Se excluyeron artículos relacionados a tratamientos. Las directrices de las principales sociedades profesionales, metanálisis, ensayos aleatorios y artículos de revisión de la literatura se priorizaron para este análisis. Se utilizaron descriptores de la salud y los medical subject headings para la selección de las palabras clave para la búsqueda de la 
información. La muestra quedó conformada por un total de 18 artículos científicos de revisión de la literatura, 5 artículos de investigación analítica.

\section{RESULTADOS}

El American College of Chest Physicians y la Society of Critical Care Medicine en el año 1991 celebraron una conferencia de consenso cuyo resultado fue el desarrollo de un nuevo conjunto de términos y definiciones, como "síndrome de respuesta inflamatoria sistémica", " sepsis "y" shock séptico "basados en parámetros clínicos y de laboratorio (1). Estas definiciones fueron acordadas y ampliamente utilizadas por la comunidad científica internacional. La frase Síndrome de respuesta inflamatoria sistémica (SIRS) se refirió al proceso inflamatorio, independientemente del tipo de insulto causante y la presencia de infección. SIRS se definió como la presencia de dos o más de los siguientes criterios: temperatura superior a $38^{\circ} \mathrm{C}$ o inferior a $36^{\circ} \mathrm{C}$; frecuencia cardíaca de más de 90 latidos / min; frecuencia respiratoria más de 20 respiraciones/min o $\mathrm{PaCO} 2$ menos de $32 \mathrm{mmHg}$; recuento anormal de glóbulos blancos (> 12 103/mL, < 4.0103 / mL, o > 10\% de formas inmaduras) (Cecconi M, 2018; Esposito S, 2017). La sepsis se definió como el SIRS en respuesta a un proceso infeccioso. La sepsis severa se definió como la sepsis asociada con disfunción orgánica, hipoperfusión o hipotensión (incluyendo acidosis láctica, oliguria y alteración aguda del estado mental). La hipotensión inducida por sepsis fue la condición de presión arterial sistólica $<90 \mathrm{mmHg}$ o una reducción de $40 \mathrm{mmHg}$ desde el inicio en ausencia de otra causa de hipotensión. El shock séptico se definió como una hipotensión persistente inducida por sepsis a pesar de la administración adecuada de líquidos intravenosos (1).

Diez años después, en el 2001 varias sociedades de cuidados intensivos europeos y norteamericanos se reunieron en una segunda conferencia de consenso para revisar estas definiciones. Ampliaron la lista de criterios de diagnóstico, pero los conceptos actuales de sepsis, sepsis grave y shock séptico se revisaron ligeramente y continuaron siendo la base de la práctica clínica y la investigación de la sepsis durante los años siguientes (1).

En 2014, la Sociedad Europea de Medicina de Cuidados Intensivos y la Sociedad de Medicina de Cuidados Críticos convocaron a un nuevo panel de expertos para actualizar las definiciones de sepsis y choque séptico (sepsis-3), que se caracterizaron por limitaciones en su especificidad y sensibilidad inadecuadas (Cecconi M, 2018). Los cambios fueron la eliminación del término sepsis severa y la eliminación del concepto de SIRS, que simplemente puede reflejar una respuesta apropiada del huésped a varias enfermedades no infecciosas (como la pancreatitis y los síndromes de reperfusión isquémica) (3). Por lo tanto, la tercera conferencia de consenso internacional, publicada del 2016, define las siguientes condiciones: la disfunción orgánica está representada por un aumento en el puntaje de la Evaluación de falla orgánica (SOFA) de 2 puntos o más; La sepsis se define como la disfunción orgánica potencialmente mortal causada por una respuesta del huésped desregulada a la infección (Cecconi M, 2018; Esposito S, 2017); 
El shock séptico se define como un subconjunto de sepsis en el que las anomalías circulatorias, celulares o metabólicas se asocian con un mayor riesgo de mortalidad (4). Los parámetros clínicos para identificar a los pacientes con shock séptico son: requerimiento de vasopresores para mantener una presión arterial media de $65 \mathrm{~mm} \mathrm{Hg}$ o mayor y un nivel de lactato sérico mayor de $2 \mathrm{mmol} / \mathrm{L}$ (> $18 \mathrm{mg} / \mathrm{dL}$ ) en ausencia de hipovolemia (5).

El mismo grupo de expertos elaboró una versión simplificada del puntaje SOFA, el puntaje SOFA rápido (quick SOFA o qSOFA), que incorpora presión arterial sistólica de $100 \mathrm{mmHg}$ o menos, frecuencia respiratoria de 22/min o mayor y alteración de la conciencia. La puntuación qSOFA se basa en criterios clínicos, pero no requiere pruebas de laboratorio, por lo tanto, proporciona una evaluación simple y rápida de pacientes con sospecha de infección que tienen más probabilidades de tener malos resultados $(4,5)$. Varios estudios recientes respaldaron el uso de las clasificaciones SOFA y qSOFA que demuestran que dichos puntajes tienen un mayor valor pronóstico que los criterios SIRS anteriores para la identificación de pacientes con mayor riesgo de mortalidad entre aquellos aferentes a los departamentos de emergencia con sospecha de infección.

\section{Reconocimiento temprano de sepsis}

El énfasis en las definiciones del grupo de trabajo sepsis-3 sobre la disfunción orgánica causada por una infección requiere que los médicos adopten un enfoque más concentrado y objetivo para la evaluación de la función del órgano. La recomendación de Sepsis-3 es utilizar una herramienta de evaluación de disfunción orgánica para identificar pacientes con sepsis. La evaluación secuencial de la insuficiencia orgánica (SOFA), más utilizada en las $\mathrm{UCl}$, es efectiva para cuantificar la gravedad de la disfunción y morbilidad de los órganos y para estimar el riesgo de mortalidad (6).

EI SOFA evalúa las siguientes funciones fisiológicas: respiración, coagulación, hepática, cardiovascular, sistema nervioso central y renal. Para calcular la puntuación SOFA de un paciente, es necesario obtener los siguientes valores de laboratorio: bilirrubina, creatinina, estudios de coagulación y gases en sangre arterial. Sin embargo, si bien estos pueden revelar disfunción orgánica, pueden no reflejar con precisión el estado de perfusión del paciente. Cuanto mayor sea el puntaje SOFA, mayor es el riesgo de morbilidad y mortalidad del paciente. (Ver el puntaje de la evaluación secuencial de insuficiencia orgánica (SOFA) $(6,7)$.

EI SOFA rápido (qSOFA), una evaluación abreviada de disfunción orgánica, se introdujo en Sepsis-3. El qSOFA se basa solo en tres variables: presión arterial sistólica, frecuencia respiratoria y mentalización. En pacientes que no son de la $\mathrm{UCl}$, el puntaje qSOFA predice un riesgo elevado de muerte y una estadía prolongada en la $\mathrm{UCl}$, pero no está diseñado para ser independiente como una advertencia temprana de sepsis o para identificar qué pacientes deben ser transferidos a la $\mathrm{UCl}$. (Consulte el puntaje de la Evaluación rápida de insuficiencia orgánica secuencial (qSOFA) $(7,8)$. 
La información impartida por los niveles séricos de lactato también puede desempeñar un papel importante en la orientación de la toma de decisiones clínicas. Un nivel de lactato sérico mayor a $2 \mathrm{mmol} / \mathrm{L}$ sugiere hipoperfusión, con niveles más altos de lactato que indican hipoperfusión más severa. La normalización de lactato en pacientes con niveles elevados de lactato sigue siendo una recomendación en las guías actuales internacionales. Dicho esto, se ha descubierto que agregar niveles de lactato sérico a los parámetros utilizados para determinar la puntuación qSOFA hace poco para mejorar su validez predictiva para la mortalidad.

Las ventajas de qSOFA son que es fácil de usar y permite a los médicos identificar pacientes en riesgo en ausencia de valores de laboratorio (6). El puntaje qSOFA no es un componente de la nueva definición de sepsis; más bien, debe alertar a los médicos sobre los pacientes que necesitan una evaluación adicional para la disfunción orgánica, lo que puede aumentar la atención para aquellos con infección previamente no reconocida o posible sepsis. Los pacientes que presentan disfunción orgánica incluso modesta asociada con infección pueden deteriorarse rápidamente; Esto subraya la importancia del reconocimiento temprano y la intervención (7). Los comentarios y recomendaciones recientes del SSC proporcionan ejemplos de cómo integrar el SOFA y el qSOFA en la evaluación de pacientes con riesgo de sepsis. Para obtener un ejemplo clínico compuesto de nuestra práctica, consulte Evaluación del riesgo de insuficiencia orgánica en pacientes con infección (8).

\section{Epidemiología}

La sepsis y el shock séptico ocurren a todas las edades. Sin embargo, existe una fuerte correlación entre la edad avanzada y la incidencia de shock séptico, con un fuerte aumento en el número de casos en pacientes mayores de 50 años. En la actualidad, la mayoría de los episodios de sepsis se observan en pacientes mayores de 60 años (Salomão R, 2019; Cecconi M, 2018; Esposito S, 2017). La edad avanzada es un factor de riesgo para adquirir infección nosocomial del torrente sanguíneo en el desarrollo de formas graves de sepsis. En general, en comparación con pacientes más jóvenes, los pacientes de edad avanzada son más susceptibles a la sepsis, tienen menos reserva fisiológica para tolerar el insulto de la infección y tienen más probabilidades de tener enfermedades subyacentes; Todos estos factores afectan negativamente la supervivencia. Además, los pacientes de edad avanzada tienen más probabilidades de tener presentaciones atípicas o inespecíficas con sepsis.

Los datos epidemiológicos han demostrado que la incidencia y la tasa de mortalidad ajustadas por edad son consistentemente mayores en los hombres; El porcentaje de pacientes varones afectados varía de $52 \%$ a $66 \%$. Sin embargo, no está claro si esta diferencia puede atribuirse a una prevalencia más alta subyacente de afecciones comórbidas o a una mayor incidencia de infección pulmonar en hombres, o si las mujeres están inherentemente protegidas contra la lesión inflamatoria que ocurre en la sepsis $(3,5)$. 
Con respecto a la etnia, un gran estudio epidemiológico mostró que el riesgo de septicemia en la población no blanca es casi el doble que, en la población blanca, con el mayor riesgo para los hombres negros. Las posibles razones de esta diferencia incluyen cuestiones relacionadas con la disminución del acceso a la atención médica y la mayor prevalencia de afecciones médicas subyacentes (9). Otro gran estudio epidemiológico relacionó la mayor incidencia en la población negra con mayores tasas de infección que requieren hospitalización y un mayor desarrollo de disfunción orgánica. En este estudio, los pacientes negros con shock séptico tuvieron una mayor incidencia de diabetes y enfermedad renal subyacente, lo que puede explicar las tasas más altas de infección (10). Sin embargo, el desarrollo de disfunción orgánica aguda fue independiente de las comorbilidades.

En resumen, los estudios epidemiológicos demuestran que la sepsis se vuelve más frecuente pero menos mortal durante las últimas décadas, al menos en países de altos ingresos, aunque la morbilidad y la mortalidad relacionadas con este síndrome siguen siendo alarmantes. Esta disminución de la mortalidad puede deberse a mejoras en los métodos de detección y a un enfoque terapéutico más agresivo, que incluye un tratamiento antimicrobiano más temprano y medidas de apoyo más efectivas.

\section{Fisiopatología}

El primer paso en el inicio de la respuesta del huésped al patógeno es la activación de las células inmunes innatas, constituidas principalmente por macrófagos, monocitos, neutrófilos y células asesinas naturales (1). Esto ocurre a través de la unión de patrones moleculares asociados a patógenos (PAMP), como las endotoxinas bacterianas y los $\beta$-glucanos fúngicos a receptores de reconocimiento de patrones específicos, en estas células $(3,5)$. Otra fuente de dicha interacción son los patrones moleculares asociados al daño (DAMP) que pueden ser material intracelular o moléculas liberadas de células huésped muertas o dañadas, como el ATP y el ADN mitocondrial. Estos se unen a receptores específicos en monocitos y macrófagos, como los receptores de peaje (TLR), los receptores de leptina de tipo C, los receptores de tipo NOD (dominio de oligomerización de unión a nucleótidos) y los receptores similares a RIG-1 (gen 1 inducible por ácido retinoico) $(11,12)$. Esto da como resultado la activación de vías de transducción de señales intracelulares que causan la transcripción y liberación de citocinas proinflamatorias como TNF $\alpha$, IL-1 e IL-6.

En la sepsis, hay una intersección entre las vías inflamatorias y hemostáticas, con la activación simultánea de las cascadas inflamatorias y de coagulación. El espectro de esta interacción puede variar desde una trombocitopenia leve hasta una coagulación intravascular diseminada fulminante (CID) (Cecconi M, 2018). La etiología de la desregulación de la coagulación en la sepsis es multifactorial. Se cree que la hipercoagulabilidad de la sepsis es impulsada por la liberación del factor tisular de las células endoteliales alteradas (otras fuentes incluyen monocitos y células polimorfonucleares) (4). De hecho, los modelos experimentales in vitro de endotoxemia y bacteriemia han demostrado una inhibición completa de la producción de 
trombina inducida por inflamación con el bloqueo del factor tisular. El factor tisular provoca la activación sistémica de la cascada de coagulación, lo que resulta en la producción de trombina, la activación de plaquetas y la formación de coágulos de plaquetas y fibrina. Estos microtrombos pueden causar defectos de perfusión locales que resultan en hipoxia tisular y disfunción orgánica.

El estado proinflamatorio inicial de sepsis a menudo es reemplazado por un estado prolongado de inmunosupresión. Hay una disminución en el número de células T (auxiliares y citotóxicas) como resultado de la apoptosis y una respuesta disminuida a las citocinas inflamatorias. Los estudios post mortem de pacientes de la $\mathrm{UCl}$ que murieron de sepsis demostraron un agotamiento global de las células T CD4 + y CD8 +, más notablemente en los órganos linfoides como el bazo $(1,5,11,12)$. Los hallazgos anteriores sugieren que el sistema inmune en un individuo séptico no puede organizar una respuesta inmune efectiva a infecciones bacterianas, virales o fúngicas secundarias.

Factores asociados a la sepsis.

Edad: El riesgo de sepsis tiene una distribución bimodal: alto en lactantes, reducido en jóvenes y aumentado nuevamente más allá de los 60 años. La edad avanzada se asocia con un mayor riesgo de sepsis; La razón puede ser que los pacientes mayores tienen una mayor predisposición a las infecciones debido a los efectos del envejecimiento en sus respuestas inmunes (12).

Sexo: existe menor incidencia de sepsis en el género femenino. Puede deberse a los efectos protectores sobre la respuesta inmune y las funciones cardiovasculares ejercidas por los estrógenos; en contraste, las hormonas sexuales masculinas deterioran la respuesta inmune mediada por células y podría explicar el mayor riesgo de sepsis en el género masculino (12).

Raza: Varios estudios analizaron el efecto de la raza sobre el riesgo de sepsis, pero sus resultados fueron discordantes (12).

Comorbilidades: El riesgo de sepsis aumenta con la presencia de al menos una de las siguientes comorbilidades: insuficiencia renal, malignidad, diabetes mellitus, enfermedad pulmonar crónica, insuficiencia cardíaca congestiva e inmunosupresión (12).

Temporada: La temporada puede ser importante en las infecciones pulmonares, que son más frecuentes en invierno y representan una causa principal de sepsis (12).

Fuente de infección: El pulmón y el abdomen son las fuentes iniciales más frecuentes de infecciones que causan sepsis (12).

Otros factores: La pobreza, la desnutrición y los niveles más bajos de educación están asociados con un mayor riesgo de sepsis. También los polimorfismos genéticos pueden interferir con la actividad de las drogas o la cinética (12).

\section{Diagnóstico microbiológico}

Se deben recolectar muestras para cultivos microbiológicos antes de administrar antibióticos en pacientes con sospecha de sepsis o shock séptico porque la terapia antimicrobiana puede 
alterar los resultados de los cultivos, lo que dificulta la identificación de los patógenos etiológicos reales $(11,12)$. Por lo tanto, en pacientes con sospecha de sepsis o shock séptico, el análisis microbiológico debe iniciarse lo antes posible, dentro de los primeros 45 minutos, para evitar demoras en el inicio de la terapia antimicrobiana. Se deben recolectar muestras de cada foco de infección sospechoso para pruebas microbiológicas en pacientes con sepsis y shock séptico: sangre, líquido cefalorraquídeo, orina, heridas, secreciones respiratorias y otros fluidos corporales.

Las técnicas estándar de microbiología basadas en cultivos a menudo producen resultados dentro de las 48-96 h, por lo tanto, la implementación de métodos de identificación rápida puede proporcionar beneficios clínicos y económicos que permiten a los médicos elegir una terapia dirigida oportunamente (13). Las técnicas de detección de patógenos moleculares están representadas por amplificación (por ejemplo, reacción en cadena de la polimerasa) o hibridación (por ejemplo, hibridación fluorescente in situ). La reacción en cadena de la polimerasa (PCR) se basa en la amplificación de secuencias diana específicas del genoma bacteriano para identificar patógenos y detectar marcadores de resistencia a los medicamentos (14).

El ensayo de hibridación fluorescente in situ (FISH) logra una identificación rápida y confiable de bacterias $u$ hongos de hemocultivos positivos mediante la hibridación con sondas de ADN marcadas con fluorescencia capaces de detectar secuencias de ARN ribosomial específicas; Las sondas de ácido nucleico peptídico (PNA) son oligómeros sintéticos con una mejor hibridación con el objetivo que las sondas FISH $(8,14)$. Estas técnicas de diagnóstico molecular requieren el crecimiento previo de microorganismos en cultivo, pero los resultados de cultivo a veces son falsos negativos en pacientes con sepsis, debido al efecto decreciente de la administración de antibióticos sobre el crecimiento microbiano.

Los patógenos etiológicos más comunes de sepsis y choque séptico son bacterias Grampositivas (Staphylococcus aureus y Staphylococcus coagulasa negativo) seguidas de Gramnegativas (Escherichia coli, Klebsiella pneumoniae, Enterobacter spp., Acinetobacter baumannii y Pseudomonas aeruginosa). La candidiasis invasiva y otros patógenos poco comunes deben considerarse en condiciones particulares, como en pacientes neutropénicos. Las bacterias resistentes a los antimicrobianos, como el Staphylococcus aureus resistente a la meticilina y los enterococos resistentes a la vancomicina, a menudo se detectan en pacientes sépticos con adquisición nosocomial de infección $(13,14)$.

\section{Biomarcadores}

La procalcitonina (PCT): Los niveles séricos normales de PCT son superiores a $0,05 \mathrm{ng} / \mathrm{ml}$ en adultos sanos y pueden usarse como un marcador de infecciones bacterianas, ya que en estas condiciones el nivel de PCT generalmente aumenta a 5000 veces, pero no aumentan en inflamaciones virales o no infecciosas. La sepsis y la inflamación sistémica pueden excluirse si la concentración plasmática de PCT es de $0.2 \mathrm{ng} / \mathrm{mL}$, mientras que los niveles plasmáticos de 
$0.5 \mathrm{ng} / \mathrm{mL}$ sugieren sepsis. La secreción de PCT comienza dentro de las primeras $2-4 \mathrm{~h}$ después del inicio de la sepsis, los niveles máximos se alcanzan a las 24-48 h. A pesar de que el nivel normal de PTC tiene un alto valor predictivo negativo, este biomarcador no puede reemplazar la evaluación clínica, por lo tanto, si los médicos sospechan que los pacientes tienen sepsis, pero los niveles de PTC permanecen bajos, los pacientes siempre deben ser tratados por sepsis (15).

El monitoreo de los niveles de procalcitonina en pacientes sépticos también puede proporcionar otra información, como la conveniencia de descontinuar la terapia antimicrobiana. En la literatura, se ha informado que varios algoritmos basados en PCT acortan la duración de la terapia con antibióticos en pacientes sépticos, y aunque su corte varía, casi todos estos algoritmos apoyan la interrupción del tratamiento antimicrobiano si el nivel de PCT es inferior a $0,5 \mathrm{ng} / \mathrm{ml}$. Sin embargo, la PCT tiene una especificidad limitada para la sepsis, ya que puede estar elevada también en pacientes no sépticos en ciertas condiciones (shock cardiogénico; pancreatitis severa; rabdomiólisis; ciertos tipos de trastornos autoinmunes) (15). La proteína $C$ reactiva (PCR) es un reactivo de fase aguda sintetizado en el hígado en respuesta a una infección y/o inflamación. Este biomarcador se usa comúnmente en la práctica clínica para detectar la presencia de procesos inflamatorios o infecciosos, pero su especificidad para el diagnóstico de sepsis es baja. La PCR no se considera el marcador ideal de sepsis porque los valores de la PCR alcanzan los niveles máximos lentamente y su disminución requiere varios días de tratamiento, por lo que la PCR no es útil para el diagnóstico precoz de pacientes sépticos (15).

El lactato es un marcador de perfusión tisular limitada o alteración del metabolismo oxidativo. Los niveles séricos altos de lactato pueden deberse a las condiciones hipóxicas asociadas a las anormalidades de perfusión y son frecuentes en pacientes críticos. Por lo tanto, independientemente de la causa de su aumento, los niveles sustanciales de lactato alto (> 4 $\mathrm{mmol} / \mathrm{L}$ ) siempre deben normalizarse en el tratamiento de pacientes con sepsis (16).

Las citocinas como biomarcadores de sepsis pueden proporcionar información adicional sobre la medición de PCT y CRP. De hecho, las citocinas (por ejemplo, IL-6) tienen un aumento temprano en la respuesta a la inflamación sistémica severa, además, los niveles altos de citocinas están correlacionados con la gravedad del proceso y con un peor resultado, pero su especificidad es baja (15).

Presepsina El subtipo de CD14 soluble (sCD14-ST), recientemente renombrado presepsina, es una glucoproteína expresada por macrófagos y monocitos como receptor de lipopolisacáridos y proteínas de unión a LPS; Tiene un papel en la respuesta inflamatoria. La concentración normal de sCD14 en suero es de 2 a $6 \mathrm{mg} / \mathrm{ml}$. Aunque la presepsina tiene una precisión diagnóstica limitada, puede indicar el desarrollo de sepsis antes que la PCT. Además, los niveles séricos de presepsina están altamente correlacionados con la gravedad de la sepsis. Por lo 
tanto, la presepsina representa un biomarcador útil para el tratamiento de pacientes sépticos, ya que permite el diagnóstico precoz y la evaluación de la gravedad (15).

sTREM-1 (Receptor de activación soluble expresado en células mieloides-1). La forma soluble del receptor desencadenante expresado en las células mieloides-1 (TREM-1) puede estar presente en varios fluidos corporales plasma, líquido cefalorraquídeo y líquido de lavado broncoalveolar) y se sobreexpresa en consecuencia la exposición a bacterias y hongos (17). suPAR (Receptor activador de plasminógeno de tipo uroquinasa soluble). El receptor activador de plasminógeno de tipo uroquinasa (UPAR) es una proteína expresada en las superficies de varios tipos de células, que desempeña un papel en la respuesta de la estimulación inflamatoria. Estudios recientes sobre suPAR informaron que tiene una especificidad moderada, de 64 a 77\%, concluyendo que su precisión diagnóstica en caso de sepsis no es superior a otros biomarcadores más comunes $(18,19)$.

Pro-ADM (pro-Adrenomedulina). Pro-ADM se puede encontrar en el plasma de pacientes con sepsis y shock séptico como consecuencia de la degradación de adrenomedulina. Un reciente estudio observacional prospectivo único informó que pro-ADM tiene la mejor precisión pronóstica para la gravedad del proceso infeccioso que cualquier otro biomarcador, incluidos PCT y PCR. De hecho, pro-ADM puede usarse para identificar pacientes con afecciones clínicas más graves que necesitan atención en la UCl y para predecir el riesgo de mortalidad hospitalaria para pacientes sépticos. Dicha información pronóstica puede ser útil para ayudar a los médicos a elegir el tratamiento más oportuno y efectivo para pacientes sépticos con mayor riesgo de peor resultado (15).

La MCP-1 (proteína quimioatrayente de monocitos 1) es una quimiocina secretada por varios tipos de células (incluidos los monocitos y las células endoteliales) y tiene efectos quimioatrayentes sobre macrófagos y linfocitos, induce la síntesis de una cascada de citocinas (IL-1 e IL-6) y la liberación de histamina por parte de los basófilos y los mastocitos, lo que contribuye significativamente a la progresión de la respuesta inflamatoria sistémica y puede desempeñar un papel en el desarrollo de la disfunción de múltiples órganos. Port lo tanto puede considerarse un biomarcador confiable, que predice con precisión el pronóstico de los pacientes con sepsis y shock séptico (15).

Inmunoglobulinas. La detección de bajos niveles de inmunoglobulinas es común en pacientes sépticos; Se han informado varias razones: disminución de la síntesis de inmunoglobulinas; hemodilución debido a la administración de fluidos o fuga capilar; aumento del catabolismo y secuestro en sitios de inflamación; consumo durante la respuesta inmune. Los niveles circulantes de inmunoglobulinas se pueden usar como un marcador del resultado: la presencia concomitante de niveles bajos de IgG1 más niveles bajos de otros isotipos de inmunoglobulinas se asocia a un aumento sustancial en el riesgo de mortalidad (15). 


\section{DISCUSIÓN}

Actualmente la comprensión de la fisiopatología compleja de la sepsis ha mejorado, y también la capacidad para definir la sepsis. Desde que Roger Bone y sus colegas sentaron las bases para la primera definición consensuada de sepsis en 1991, ha habido avances significativos en la patobiología de la de la enfermedad. Tenemos una mejor comprensión de la biología celular, bioquímica, inmunología y la morfología, así como los cambios en la circulación y la función de los órganos (1). Esta comprensión ha llevado a los cambios en la definición de sepsis. Esto también ha contribuido a un mejor manejo de la sepsis que conduce a cambios en la epidemiología de la sepsis.

Según Sepsis-3, la sepsis se define como una disfunción orgánica grave, potencialmente mortal, causada por una respuesta inadecuada o desregulada del huésped a la infección. Por lo tanto, el término "sepsis severa" se vuelve redundante y ya no debe usarse. Como criterio de cabecera para identificar a pacientes adultos con sospecha de infección fuera de la UCl que probablemente tengan malos resultados, Sepsis-3 propuso el qSOFA: alteración de la concentración, presión arterial sistólica de $100 \mathrm{~mm} \mathrm{Hg}$ o menos y frecuencia respiratoria de $22 /$ min o más $(14,19)$. La presencia de al menos 2 variables sugiere un paciente con alto riesgo de resultados desfavorables, como la muerte hospitalaria o una estadía prolongada en la $\mathrm{UCl}$. Claramente, la sepsis ya no podría caracterizarse únicamente como una respuesta inflamatoria sistémica desencadenada por una infección, y el concepto revisado - Sepsis 3 - ha redefinido la sepsis como una disfunción orgánica potencialmente mortal causada por una respuesta desregulada del huésped a la infección. Este concepto ha actualizado la complejidad de la respuesta del huésped y la consiguiente presentación clínica de los pacientes, abriendo caminos para nuevas estrategias de tratamiento.

Russell J (13), menciona que la incidencia de sepsis ha estado creciendo en las últimas décadas, por razones que probablemente incluyen lo siguiente: una población cada vez más anciana, mayor reconocimiento de la enfermedad, mayor rendimiento de procedimientos invasivos y trasplante de órganos, mayor uso de agentes inmunosupresores y quimioterapia, mayor uso de líneas y dispositivos permanentes y un aumento de enfermedades crónicas como la enfermedad renal en etapa terminal (ERCT) y la infección por VIH.

La patogenia de la sepsis es compleja e involucra múltiples aspectos de la interacción entre los microorganismos infectantes y el huésped. El reconocimiento de los patógenos y la activación celular resultante son fundamentales para el control de infecciones. Paradójicamente, la respuesta inflamatoria del huésped también es el sustrato de los cambios fisiopatológicos en la sepsis

Estudios recientes han abordado este importante tema. En un estudio multicéntrico en Australia y Nueva Zelanda que evaluó alrededor de 180 mil pacientes ingresados en $182 \mathrm{UCl}$, la discriminación de la mortalidad hospitalaria fue significativamente mayor usando SOFA 
(AUROC, 0.753 [IC del 99\%, 0.750-0.757]) que cualquiera de los criterios de SIRS (AUROC, 0.589 [IC 99\%, 0.585-0.593]) o qSOFA (AUROC, 0.607 [IC 99\%, 0.603-0.611]) (20). Los autores concluyeron que los criterios de SIRS no proporcionaron un uso predictivo adicional para la mortalidad o una estadía prolongada en la UCl más allá de la alcanzada con SOFA y que la puntuación qSOFA tenía poco valor predictivo adicional sobre los criterios de SIRS entre los pacientes ingresados en la UCI con sospecha de infección.

La validez de los criterios de Sepsis-3 fue evaluada por Freund y sus compañeros de trabajo en una cohorte prospectiva de pacientes que acudieron al servicio de urgencias con sospecha de infección. Los rendimientos de qSOFA y SOFA para predecir la mortalidad hospitalaria (punto final primario) y el ingreso a la $\mathrm{UCl}$, la duración de la estadía en la $\mathrm{UCl}$ de más de $72 \mathrm{~h}$, y un compuesto de muerte o estadía en la $\mathrm{UCl}$ de más de $72 \mathrm{~h}$ (final secundario puntos) se compararon con los de SIRS y la combinación de SIRS y un nivel de lactato en sangre superior a $2 \mathrm{mmol} / \mathrm{L}(18 \mathrm{mg} / \mathrm{dL})$ (11). El uso de qSOFA resultó en una mayor precisión pronóstica para la mortalidad hospitalaria que SIRS o sepsis severa. Estos resultados sugieren que qSOFA puede ser útil para la selección de pacientes con sospecha de infección y riesgo de resultados desfavorables fuera de la $\mathrm{UCl}$, mientras que SOFA tendría una mejor precisión para aquellos con sepsis que ya están en la UCl.

La inclusión de biomarcadores en la batería de estudios para sepsis y shock séptico es importante, ya que ayudan a complementar el diagnóstico y pronóstico. Según un metaanálisis desarrollado por Aksaray S, el valor diagnóstico del STREM-1 es limitado (con una sensibilidad del $79 \%$ y una especificidad del $80 \%$ ), por lo que no puede usarse solo para confirmar al sospechoso de sepsis (6). Estudios recientes sobre suPAR informaron que tiene una especificidad moderada, de 64 a 77\%, concluyendo que su precisión diagnóstica en caso de sepsis no es superior a otros biomarcadores más comunes $(15,16,17)$. Incluso, un estudio que mostró que un recuento bajo de linfocitos al inicio de la sepsis (día 4 del diagnóstico) es predictivo de mortalidad a los 28 días y al año, se ha postulado que la linfopenia temprana puede servir como un biomarcador para la inmunosupresión en la sepsis (20).

Existe una continuidad clínica de gravedad, desde sepsis hasta shock séptico y síndrome de disfunción orgánica múltiple. En un estudio que evaluó a 2527 pacientes de la unidad de cuidados intensivos con síndrome de respuesta inflamatoria sistémica, el $26 \%$ desarrolló sepsis, el $18 \%$ desarrolló sepsis grave y el $4 \%$ desarrolló shock séptico. La incidencia de resultados positivos en el hemocultivo fue del $17 \%$ en pacientes con sepsis y del $69 \%$ en pacientes con shock séptico (23). El diagnóstico de shock séptico requiere características de SIRS (cambios mentales, hiperventilación, hemodinámica distributiva, hipertermia o hipotermia, o glóbulos blancos [leucocitos] reducidos, elevados o desplazados a la izquierda) además de una fuente potencial de infección. Siempre que un paciente se presenta con shock, se debe formular un diagnóstico de trabajo temprano, se debe establecer un enfoque para la reanimación urgente y se deben tomar medidas para confirmar el diagnóstico de trabajo. 
Un paciente con sepsis que ingresa en la $\mathrm{UCl}$ debe ser monitoreado cuidadosamente para facilitar la prevención y el tratamiento de las complicaciones infecciosas que pueden perpetuar SIRS o desencadenar sepsis recurrente después de la mejoría inicial. Tales complicaciones incluyen sinusitis, infección del tracto urinario, urosepsis, infecciones relacionadas con el catéter intravascular, colecistitis acalculosa y translocación de bacterias o endotoxinas desde el intestino. Varias de estas dolencias pueden no manifestarse clínicamente; en consecuencia, un alto índice de sospecha es crucial para el diagnóstico y tratamiento tempranos.

\section{CONCLUSIONES}

La sepsis sigue siendo una carga importante para los sistemas de salud en todo el mundo. Sin embargo, los avances realizados en la comprensión de su patogénesis y los esfuerzos extensivos para enmarcar las pautas para su manejo efectivo en los últimos 20 años superan todo lo que se ha hecho antes. En la actualidad, estamos en una transición entre la definición anterior, que guió el manejo clínico y apoyó intervenciones exitosas, y una nueva definición que ha introducido avances incuestionables al incorporar el conocimiento actual en fisiopatología y proporcionar criterios de diagnóstico más sólidos, pero aún plantea discusión sobre el uso de criterios para la identificación versus el pronóstico del paciente séptico.

Las definiciones revisadas de sepsis y shock séptico se han publicado recientemente y se han adoptado ampliamente tanto para la investigación como para la práctica clínica. A pesar de los avances en el manejo clínico, incluidos los hallazgos de nuevos biomarcadores con valor diagnóstico y pronóstico, la sepsis y el shock séptico siguen siendo una causa principal de ingreso a la unidad de cuidados intensivos en todo el mundo, aunque las tasas de mortalidad asociadas a estos síndromes disminuyeron en los países de altos ingresos durante los últimos años.

Los nuevos datos emergentes nos ayudarán a delinear un mejor algoritmo para la identificación y atención de pacientes sépticos, tanto en el amplio alcance de intervenciones bien organizadas y planificadas como de manera individual.

\section{REFERENCIAS}

1. Salomão R. Sepsis: evolving concepts and challenges. Braz J Med Biol Res. Vol. 52(4):e8595. doi:10.1590/1414-431X20198595. 2019.

2. Cecconi M. Sepsis and septic shock. Lancet. Vol. 392(10141):75-87. doi:10.1016/S01406736(18)30696-2. 2018.

3. Esposito S. Sepsis and septic shock: New definitions, new diagnostic and therapeutic approaches. J Glob Antimicrob Resist. Vol. 10:204-212. doi:10.1016/j.jgar.2017.06.013. 2017. 
4. Gyawali B. Sepsis: The evolution in definition, pathophysiology, and management. SAGE Open Med. Vol. 7:2050312119835043. doi:10.1177/2050312119835043. 2019.

5. Kim M. An Update on Sepsis Biomarkers. Infect Chemother. Vol. 52(1):1-18. doi:10.3947/ic.2020.52.1.1. 2020.

6. Gole A. Prognostic accuracy of SOFA score, SIRS criteria, NEWS and MEWS scores for inhospital mortality among adults admitted to ICU with suspected sepsis. J Assoc Physicians India. 2020;68(1):87. 2020.

7. Marik P. SIRS, qSOFA and new sepsis definition. J Thorac Dis. Vol. 9(4):943-945. doi:10.21037/jtd.2017.03.125. 2017.

8. Andaluz D. SIRS, qSOFA, and organ failure for assessing sepsis at the emergency department. J Thorac Dis. Vol. 9(6):1459-1462. doi:10.21037/jtd.2017.05.36. 2017.

9. Martin G. La epidemiología de la sepsis en los Estados Unidos desde 1979 hasta 2000. N Engl J Med. Vol. 348 (16): 1546-54. 2003.

10. Mayr et al. Tasa de infección y riesgo de disfunción orgánica aguda como explicaciones de las diferencias raciales en la sepsis severa. JAMA. Vol. 303 (24): 2495-2503. 2010.

11. Freund et al. Precisión pronóstica de los criterios de sepsis-3 para la mortalidad hospitalaria entre pacientes con sospecha de infección que acuden al servicio de urgencias. JAMA. Vol. 317 : 301-308. doi: 10.1001 / jama.2016.20329. 2017.

12. Makic M. Managing Sepsis and Septic Shock: Current Guidelines and Definitions. Am J Nurs. Vol.118(2):34-39. doi:10.1097/01.NAJ.0000530223.33211.f5. 2018.

13. Russell J. Pathophysiology of Septic Shock. Crit Care Clin. Vol. 34(1):43-61. doi:10.1016/j.ccc.2017.08.005. 2018.

14. Peach B. Implications of the new sepsis definition on research and practice. J Crit Care. Vol. 38:259-262. doi:10.1016/j.jcrc.2016.11.032. 2017.

15. Singer M. Biomarkers for sepsis - past, present and future. Qatar Med J. Vol. 2019(2):8. doi:10.5339/qmj.2019.qccc.8. 2019.

16. Casserly et al. Lactate measurements in sepsis-induced tissue hypoperfusion: results from the Surviving Sepsis Campaign database. Crit Care Med. Vol. 43(3):567-573. doi:10.1097/CCM.0000000000000742. 2015.

17. Aksaray S. Diagnostic value of STREM-1 and procalcitonin levels in the early diagnosis of sepsis. North Clin Istanb. Vol. 3(3):175-182. doi:10.14744/nci.2016.26023. 2017.

18. Donadello K. suPAR as a prognostic biomarker in sepsis. BMC Med. Vol. 10:2. doi:10.1186/1741-7015-10-2. 2012. 
19. Huang et al. The Diagnostic and Prognostic Value of suPAR in Patients with Sepsis: A Systematic Review and Meta-Analysis. SHOCK. Vol. 53 (4): 416-425. doi: 10.1097/SHK.0000000000001434. 2020.

20. Singer et al. The Third International Consensus Definitions for Sepsis and Septic Shock (Sepsis-3). JAMA. Vol. 315(8):801-810. doi:10.1001/jama.2016.0287. 2016.

21. Raith et al. Precisión pronóstica de la puntuación SOFA, los criterios SIRS y la puntuación qSOFA para la mortalidad hospitalaria entre adultos con sospecha de infección ingresados en la unidad de cuidados intensivos. JAMA. 317 : 290-300. doi: 10.1001 / jama.2016. 2017.

22. Seymour et al. Assessment of Clinical Criteria for Sepsis: For the Third International Consensus Definitions for Sepsis and Septic Shock (Sepsis-3). JAMA. Vol. 315(8):762-774. doi:10.1001/jama.2016.0288. 2016. 